\title{
Rester ou partir ? Les déterminants des flux d'émigration récents depuis la Suisse
}

Stay or Leave? Deciding Factors in the Recent Emigration Flow from Switzerland Los determinantes de los flujos recientes de emigración desde Suiza

Juliette Fioretta et Philippe Wanner

\section{(2) OpenEdition}

Journals

\section{Édition électronique}

URL : https://journals.openedition.org/remi/8532

DOI : $10.4000 /$ remi.8532

ISSN : $1777-5418$

Éditeur

Université de Poitiers

Édition imprimée

Date de publication : 1 mars 2017

Pagination : 111-131

ISBN : 979-10-90426-30-6

ISSN : 0765-0752

\section{Référence électronique}

Juliette Fioretta et Philippe Wanner, «Rester ou partir ? Les déterminants des flux d'émigration récents depuis la Suisse », Revue européenne des migrations internationales [En ligne], vol. $33-\mathrm{n}^{\circ} 1$ 2017, mis en ligne le 01 mars 2019, consulté le 14 avril 2022. URL : http://journals.openedition.org/ remi/8532 ; DOl : https://doi.org/10.4000/remi.8532 


\section{Rester ou partir ? Les déterminants des flux d'émigration récents depuis la Suisse}

\section{Juliette Fioretta ${ }^{1}$ et Philippe Wanner ${ }^{2}$}

\section{Introduction}

L'émigration, qu'elle soit temporaire ou définitive, qu'elle fasse référence à un retour d'immigrants ou à une première migration de natifs, représente de nombreux défis pour la société. Ceux-ci sont liés, suivant les contextes, à la perte de compétences professionnelles, au dépeuplement de certaines régions périphériques ou à l'affaiblissement des finances publiques. Ceci est encore plus le cas lorsque l'émigration est sélective, en d'autres termes lorsqu'elle concerne des personnes hautement qualifiées, des élites ou des contribuables aisés. Cependant, malgré l'importance de l'émigration, la majorité des études portant sur le thème des mouvements internationaux de personnes dans les pays industrialisés délaissent ce phénomène pour s'intéresser à l'autre facette des flux migratoires, à savoir les mouvements d'entrée.

La Suisse dénombre chaque année plus de 100000 départs en direction de l'étranger, un chiffre en augmentation depuis 2003 (année où le nombre d'émigrations atteignait 76800$)^{3}$. Avec un taux de treize mouvements émigratoires pour 1000 habitants en 2013, la Suisse se positionne en troisième position des pays d'Europe occidentale derrière le Luxembourg (20\%) et I'Islande (14\%o), mais largement devant des pays de taille comparable, tels l'Autriche et les

1 Institut de démographie et de socioéconomie, Université de Genève, Pont d'Arve 40, 1211 Genève 4, Suisse ; juliette.fioretta@unige.ch

Cette étude a été réalisée dans le cadre du projet 100017_152566 intitulé « impact économique et social de l'émigration et des mouvements migratoires en Suisse " et financé par le Fonds National Suisse de la Recherche Scientifique.

2 Professeur, Institut de démographie et de socioéconomie, Université de Genève, Pont d'Arve 40, 1211 Genève 4, Suisse ; philippe.wanner@unige.ch

3 Ces chiffres font référence à la population résidente permanente, définie pour les étrangers comme la population titulaire d'un permis de séjour d'une durée égale ou supérieure à une année. Ils excluent donc les personnes effectuant un séjour de courte durée. Le nombre le plus élevé de départs observés au cours d'une année, depuis l'établissement des statistiques sur la migration internationale en 1981, fut enregistré en 1992, avec un effectif de 117034 départs, expliqués principalement par une conjoncture économique défavorable. Ces chiffres sont consultables sur le site de l'Office fédéral de la statistique (www.bfs.admin.ch). 
Pays-Bas $(6 \%)^{4}$. L'importance de l'émigration surprend, alors que la conjoncture économique dans le pays et le marché du travail présentent une situation favorable en comparaison internationale. La Suisse bénéficie de conditions sur le marché du travail ou fiscales compétitives en comparaison européenne. Vu le nombre de départs, il est donc important de comprendre qui sont les résidents quittant le pays, et plus particulièrement quels sont les facteurs intervenant dans le choix d'effectuer une émigration.

Le développement de nouvelles données statistiques permet désormais de mesurer le rôle exact de différentes dimensions socioéconomiques sur le fait d'émigrer. Cet article exploite ces nouvelles données pour la période 2011 à 2013, en vue de vérifier quels facteurs individuels influent la probabilité de quitter la Suisse. II poursuit et précise les travaux publiés par Pecoraro (2012) qui, utilisant les recensements 1990 et 2000, avait mis en évidence l'existence de différents déterminants socioéconomiques à l'émigration. Les années 2011 à 2013 ont enregistré des flux migratoires situés dans la moyenne des dix dernières années. Elles sont donc représentatives du régime migratoire actuel, caractérisé par la libre circulation des personnes.

Cet article se focalise sur les facteurs individuels intervenant sur la probabilité de départ sans s'intéresser au pays de domicile après le mouvement émigratoire. Compte tenu des spécificités liées aux différents types d'émigrations (départ du pays de naissance, déménagement dans un état tiers ou retour dans son pays d'origine), l'analyse distinguera les natifs et les immigrés. Elle visera, dans un premier temps, à identifier les facteurs modifiant la probabilité de quitter la Suisse. Elle confrontera ensuite les résultats ainsi obtenus aux théories expliquant l'émigration afin de déterminer si l'émigration de la Suisse présente un caractère particulier où si l'on y retrouve des traits communs à d'autres pays industrialisés.

\section{Données et méthodes}

Les analyses reposent sur les données de trois relevés structurels suisses (2010 à 2012). Chaque relevé interroge plus de 200000 personnes âgées de quinze ans et plus par questionnaire écrit ou en ligne en fin d'année (date de référence : 31 décembre) et fait partie intégrante du nouveau système de recensement fédéral de la population. La population interrogée comprend des Suisses et des étrangers résidents permanents, c'est-à-dire vivant en Suisse depuis au moins une année ou bénéficiaires d'un permis de séjour annuel (permis B) ou d'établissement (permis $\mathrm{C}$ ). Les questions posées dans le cadre de cette enquête se rapportent entre autres à la situation familiale, au niveau de formation, à la position sur le marché du travail et au statut migratoire.

Les données des relevés structurels ont été appariées avec celles du registre harmonisé des habitants, qui a été introduit en Suisse en 2010. Ce registre exhaustif informe sur l'éventuel départ à l'étranger des personnes résidant en Suisse. L'appariement, reposant sur le numéro AVS (numéro d'identification personnel anonyme entré en vigueur à la fin de la première décennie du

4 Source : propres calculs selon données Eurostat. 
XXle siècle), permet dès lors d'identifier le départ des résidents ayant répondu au relevé structurel ${ }^{5}$.

Les analyses prennent en compte 822340 individus ayant participé aux relevés structurels 2010, 2011 ou 2012. Seuls ceux ayant déclaré un lieu de naissance ont été retenus. Pour chaque individu, le statut émigratoire a été déterminé en se référant au registre des habitants pour les années 2011 à 2013. Au total, 12237 personnes ont quitté la Suisse avant la fin 2013. Le motif du départ n'est pas documenté, de même que le type (départ définitif) et la durée du séjour à l'étranger. L'émigration peut se référer à différentes motivations (stage, retour dans le pays, séjour professionnel à l'étranger, etc.) qui ne sont pas connues. Les analyses distinguent d'une manière systématique les personnes nées en Suisse d'une part et celles nées à l'étranger de l'autre. Ces deux groupes répondent en effet à des motivations et logiques migratoires différentes et montrent également des niveaux d'émigration distincts.

Alors que le registre des habitants fournit le statut migratoire, le relevé structurel indique le statut économique, social et familial de l'individu à la date de l'enquête. Les variables supposées influencer étroitement la probabilité de quitter la Suisse ont été retenues. La distribution des modalités de ces variables figure en annexe. Ces variables sont les suivantes:

- La nationalité, regroupée en six catégories : Suisse ; pays européens traditionnellement fournisseurs de main-d'œuvre (Allemagne, Italie, France, Espagne et Portugal) ; autres pays membres de I'UE/AELE ${ }^{6}$; autres pays européens ${ }^{7}$; pays de I'OCDE non européens ${ }^{8}$; reste du monde.

- Le statut migratoire, en trois catégories : Suisse, primo-migrant et enfant de migrant. Font partie de cette dernière catégorie les personnes nées en Suisse : de nationalité suisse depuis la naissance et dont les deux parents sont nés à l'étranger ; naturalisés au cours de leur vie, dont au moins un des deux parents est né à l'étranger ; de nationalité étrangère ; ainsi que les personnes nées à l'étranger et étant arrivées en Suisse avant l'âge de dix ans.

- La durée de séjour en Suisse, issue du registre des habitants. Pour les personnes nées à l'étranger, mais pour qui la date de la dernière arrivée en Suisse est manquante, l'information a été complétée en utilisant des informations issues du registre central des étrangers (registre SYMIC) ${ }^{9}$. Ce registre informe sur la population de nationalité étrangère au bénéfice d'un permis de séjour et a été apparié avec le relevé structurel à I'aide du numéro AVS (Steiner et Wanner, 2015). La durée de séjour a été codée en quatre catégories : en Suisse depuis moins d'une année ; depuis un à moins de cinq ans; depuis cinq à moins de dix ans; et depuis plus de dix ans.

5 Voir Steiner et Wanner (2015) pour une description détaillée des procédures d'appariement.

6 Belgique, Bulgarie, Danemark, Finlande, Grèce, Royaume-Uni, Irlande, Islande, Liechtenstein, Luxembourg, Malte, Pays-Bas, Norvège, Autriche, Pologne, Roumanie, Suède, Hongrie, Chypre, Slovaquie, République tchèque, Croatie, Slovénie, Estonie, Lettonie, Lituanie.

7 Albanie, Monaco, Saint-Marin, Turquie, Serbie, Bosnie et Herzégovine, Monténégro, Macédoine, Kosovo, Moldova, Russie, Ukraine, Belarus.

8 Chili, Canada, Mexique, États-Unis, Israël, Japon, Corée (Nord), Australie, NouvelleZélande.

9 Ce complément a pu être réalisé pour les personnes déjà présentes en Suisse au 31 décembre 2010. 
- La présence d'un épisode migratoire est construite, pour les personnes nées en Suisse, en recourant à la date de l'arrivée en Suisse ${ }^{10}$.

- Les variables socioéconomiques font référence au capital humain et à l'insertion sur le marché du travail (statut d'activité). Le capital humain est appréhendé par le plus haut niveau de formation atteint par la personne, classé en trois catégories : Secondaire I (aucune école obligatoire ou jusqu'à sept ans au maximum, ou école obligatoire - huit ou neuf ans -, ou dixième année de préapprentissage ou autres passerelles proposées) ; Secondaire II (formation professionnelle initiale - préapprentissage, apprentissage de deux à quatre ans ou école professionnelle à plein temps, diplôme de commerce, ateliers d'apprentissage et formation similaire - ou école de culture générale - maturité gymnasiale, maturité professionnelle ou spécialisée, école normale, école de degré diplôme, école des transports ou formation équivalente) ; Tertiaire (formation supérieure spécialisée et professionnelle avec brevet fédéral, diplôme ou maîtrise, école technique, école supérieure, ETS, ESCEA, ESAA, ESEF ou formation équivalente ; université, EPF, haute école pédagogique, haute école spécialisée).

- Le statut d'activité est distribué en six modalités : actif (à temps partiel ou temps plein) ; au chômage ; en formation ; sans activité (au foyer) ; rentier ; " autre, sans activité professionnelle ". Le statut d'activité est autodéclaré par les personnes enquêtées et il est pour cette raison difficile de définir un profil exact des personnes se déclarant sans activité, sans pour autant être rentières ou au foyer.

- La situation personnelle au moment de l'enquête est déterminée par le type de propriété du logement, en deux catégories ; propriétaire (propriété par étage ou de la maison d'habitation) ou locataire (locataire, sous-locataire, coopérateur, occupant d'un logement de service). Par ailleurs, la composition du ménage est considérée en trois modalités : ménage d'une personne ou ménage non familial de plusieurs personnes; ménage familial sans enfant; ménage familial avec enfants (couples avec enfants, familles monoparentales, ménages multifamiliaux).

- Finalement, le lieu de résidence est pris en compte selon deux variables. La première fait référence à la proximité de la frontière et distingue les agglomérations frontalières (Genève, Bâle, Schaffhouse, Mendrisio et Come) du reste du territoire. La seconde tient compte du type de commune (urbain ou rural).

Les facteurs associés à l'émigration depuis la Suisse peuvent être décrits par le calcul de probabilités ou quotients d'émigration, calculés en divisant le nombre de départs par la population rencontrée au relevé structurel. La durée d'observation variant entre un (pour les personnes ayant participé au relevé structurel du 31 décembre 2012) et trois ans (pour celles ayant participé au relevé structurel du 31 décembre 2010), on tient compte de la variation de la durée d'observation et on présente des probabilités de départ se référant à une période annuelle. Les probabilités sont pondérées, afin d'être représentatives de l'ensemble de la population âgée de quinze ans et plus résidant en Suisse. La pondération a été calculée et fournie par l'Office fédéral de la statistique qui est en charge du relevé structurel.

10 Les natifs de la Suisse ayant effectué un séjour à l'étranger ont une indication de la date d'arrivée en Suisse dans le registre des habitants. 
Des modèles de régression logistique permettent par ailleurs de mieux déterminer si certaines caractéristiques peuvent prédire l'émigration, exprimée sous la forme d'une variable binaire (quitte la Suisse ou non). Les modèles de régression mesurent en effet des probabilités de départ pour une variable (par exemple, le sexe), exprimées par rapport à une catégorie ou modalité de référence (par exemple, les hommes), en tenant compte des effets des autres variables incluses dans le modèle (âge, nationalité, etc.). Les effets nets, exprimés sous la forme de odds ratios, sont ainsi disponibles (Cox et Snell, 1989). Un odds ratio supérieur à l'unité indique une probabilité plus élevée d'émigration pour la catégorie étudiée, comparativement à la catégorie de référence. Les modèles ont été calculés séparément pour la population née en Suisse et celle née à l'étranger. Pour cette dernière, on distingue en outre deux modèles, en fonction de la nationalité, afin de mieux identifier des effets de variables spécifiques intervenant différemment sur la probabilité de départ des Suisses et des étrangers. Nous avons conservé dans les analyses uniquement les individus n'ayant pas de données manquantes sur l'ensemble des variables prises en compte, après avoir vérifié la robustesse des résultats ${ }^{11}$. Trois modèles sont donc proposés, incluant des variables communes (par exemple, sexe ou groupe d'âge) et des variables spécifiques (par exemple, nationalité pour les étrangers ou durée de séjour en Suisse pour les migrants).

\section{Résultats}

Le tableau 1 présente les quotients d'émigration et les effectifs, en fonction du lieu de naissance et des différentes caractéristiques individuelles, ainsi que les résultats des régressions logistiques. Le degré de signification des résultats est aussi indiqué. Les probabilités d'émigrer varient d'une manière importante en fonction du lieu de naissance : on dénombre en effet approximativement un départ annuel pour 350 personnes nées en Suisse, contre un pour cinquante personnes nées à l'étranger. Ainsi que l'indiquent les résultats des régressions logistiques, les facteurs influençant la migration sont relativement similaires pour les trois groupes distingués (nés en Suisse, nés à l'étranger et de nationalité suisse, nés à l'étranger et de nationalité étrangère), les valeurs des odds ratios étant généralement proches, avec cependant de faibles variations qui sont passées en revue ci-dessous.

11 Les coefficients et leur significativité sont semblables entre les modèles prenant ou ne prenant pas en compte les valeurs manquantes. 
Tableau 1 : Probabilités annuelles d'émigrer et odds ratios selon le lieu de naissance et la nationalité, Suisse, 2011-2013

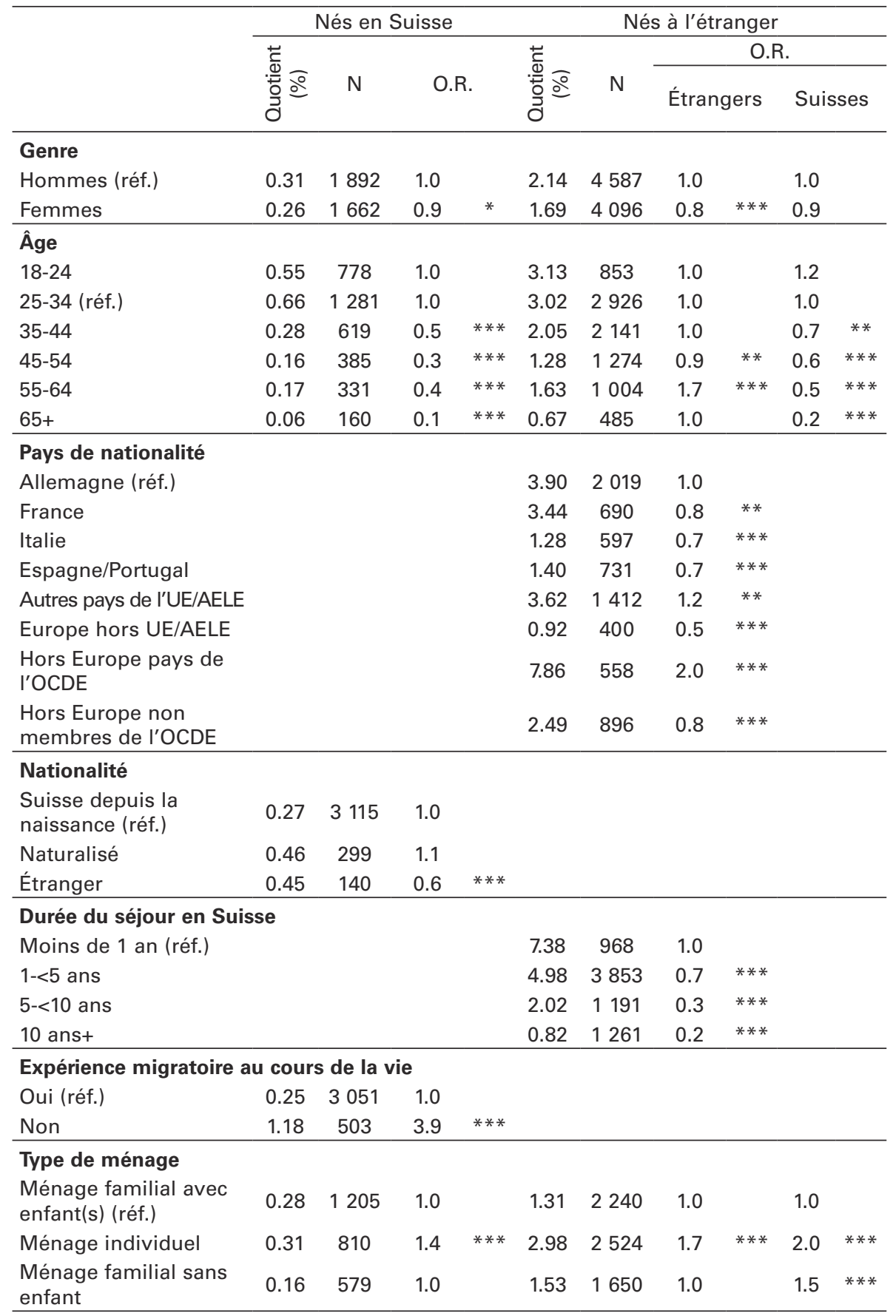




\begin{tabular}{|c|c|c|c|c|c|c|c|c|c|c|}
\hline \multicolumn{11}{|l|}{ Statut d'activité } \\
\hline Actif (réf.) & 0.29 & 2457 & 1.0 & & 1.85 & 5446 & 1.0 & & 1.0 & \\
\hline Au chômage & 0.91 & 224 & 2.2 & $* * *$ & 3.15 & 747 & 1.6 & $* * *$ & 2.4 & $* * *$ \\
\hline Étudiant & 0.82 & 358 & 1.9 & $* * *$ & 6.0 & 703 & 2.7 & $* * *$ & 2.1 & $* * *$ \\
\hline Au foyer & 0.2 & 107 & 1.2 & & 2.42 & 792 & 2.0 & $* * *$ & 2.1 & $* * *$ \\
\hline AVS/Al & 0.08 & 243 & 1.1 & & 0.76 & 682 & 1.2 & & 1.1 & \\
\hline Autre & 1.52 & 155 & 3.7 & $* * *$ & 3.55 & 272 & 1.9 & $* * *$ & 2.9 & $* * *$ \\
\hline \multicolumn{11}{|l|}{ Niveau de formation } \\
\hline Tertiaire (réf.) & 0.45 & 1501 & 1.0 & & 3.34 & 4630 & 1.0 & & 1.0 & \\
\hline Secondaire I & 0.17 & 360 & 0.4 & $* * *$ & 1.12 & 1729 & 0.5 & $* * *$ & 0.6 & $* * *$ \\
\hline Secondaire II & 0.24 & 1658 & 0.6 & $* * *$ & 1.42 & 2029 & 0.6 & $* * *$ & 0.6 & $* * *$ \\
\hline \multicolumn{11}{|l|}{ Proximité de la frontière } \\
\hline Autre agglomération (réf.) & 0.25 & 2796 & 1.0 & & 1.83 & 6690 & 1.0 & & 1.0 & \\
\hline $\begin{array}{l}\text { Agglomération } \\
\text { frontalière }\end{array}$ & 0.51 & 758 & 1.6 & $* * *$ & 2.19 & 1993 & 1.1 & & 1.5 & $* * *$ \\
\hline \multicolumn{11}{|l|}{ Lieu de résidence } \\
\hline Commune urbaine (réf.) & 0.32 & 2882 & 1.0 & & 1.96 & 7564 & 1.0 & & 1.0 & \\
\hline Commune rurale & 0.19 & 672 & 0.8 & $* * *$ & 1.62 & 1119 & 0.9 & * & 1.2 & \\
\hline \multicolumn{11}{|l|}{ Statut du logement } \\
\hline Locataire (réf.) & & & 1.0 & & & & 1.0 & & 1.0 & \\
\hline Propriétaire & & & 0.5 & $* * *$ & & & 0.5 & $* * *$ & 0.5 & $* * *$ \\
\hline Constante & & & 0.0 & $* * *$ & & & 0.2 & $* * *$ & 0.0 & $* * *$ \\
\hline $\mathrm{N}$ & & & 481 & 629 & & & 1130 & & & 367 \\
\hline Nombre d'émigrations & & & 2538 & & & & 5189 & & 943 & \\
\hline Chi carré & & & 2798 & $* * *$ & & & 5705 & $* * *$ & 638 & $* * *$ \\
\hline $\begin{array}{l}\text { Pseudo R² de } \\
\text { Nagelkerke }\end{array}$ & & & 0.1 & & & & 0.2 & & 0.1 & \\
\hline
\end{tabular}

$$
{ }^{*} p<0.05 ;{ }^{* *} p<0.01 ;{ }^{* * *} p<0.001
$$

Source : Relevés structurels 2010-2012 et registre des habitants 2011-2013 ${ }^{12}$.

Exemple de lecture : Quotient : parmi la population née à l'étranger de nationalité étrangère et de sexe masculin, la probabilité de quitter la Suisse est de 2,14\%, indiquant que pour 100 hommes présents en début d'année, deux ont quitté la Suisse avant la fin de l'année. Odds ratio : comparativement à cette valeur et après prise en compte des autres variables incluses dans le modèle, la valeur de la cote pour une femme née à l'étranger et de nationalité étrangère de quitter la Suisse est réduite de $20 \%$.

12 Sauf mention contraire, tous les tableaux de l'article proviennent des relevés structurels 2010-2012 et du registre des habitants 2011-2013. 
D'une manière générale, les hommes présentent une probabilité d'émigrer légèrement supérieure à celle des femmes, un résultat significatif pour les étrangers nés à l'étranger et les natifs de la Suisse uniquement, mais non significatif pour les Suisses nés à l'étranger. La mobilité accrue des hommes, mise en évidence par d'autres auteurs (Nekby, 2006 ; Reagan et Olsen, 2000), est relativement modeste après prise en compte des autres facteurs : I'odds ratio des femmes comparativement aux hommes est compris entre 0.8 (étrangers nés à l'étranger) et 0.9 (natifs de la Suisse), signifiant un risque diminué de 10 à $20 \%$.

Cette différence de genre pourrait s'expliquer par le rôle de la migration sur la position des hommes et des femmes dans la société, selon Bachmeier et al. (2013). Pour ces auteurs, les femmes immigrant vers des pays industrialisés arrivent dans un contexte qui présente généralement une plus grande orientation vers l'égalité de genre que celui de leur pays d'origine, d'où un gain dans leur statut et une moindre propension à émigrer. Au contraire, les hommes dans cette situation font souvent l'expérience d'une perte de statut, tant au niveau des rapports de genre que de leur statut social dans la société d'accueil et auront donc une plus grande propension à émigrer (migration de retour). Cette explication, quoique intéressante d'un point de vue théorique, ne devrait concerner qu'une minorité des migrants dans le cas suisse : I'essentiel de la migration est en effet issu de pays voisins présentant des caractéristiques proches en termes d'égalité de genre. En consultant le rapport des quotients d'émigration entre hommes et femmes (Tableau 2), on s'aperçoit en effet que celui-ci est le plus élevé parmi les ressortissants des pays non européens et qu'il est plus faible parmi les pays voisins et de I'UE/AELE, Allemagne exceptée. II est probable que les opportunités offertes aux femmes dans la société suisse puissent jouer un rôle, mais évidemment d'autres facteurs interviennent aussi.

Tableau 2 : Probabilités annuelles d'émigrer selon le genre et le pays de nationalité pour les personnes nées à l'étranger, Suisse et ratio hommes/femmes, 2011-2013

\begin{tabular}{|c|c|c|c|c|c|}
\hline & \multicolumn{2}{|c|}{ Hommes } & \multicolumn{2}{|c|}{ Femmes } & \multirow[b]{2}{*}{ Ratio } \\
\hline & Quotient (\%) & $\mathrm{N}$ & Quotient (\%) & $\mathrm{N}$ & \\
\hline Suisse & 0.84 & 594 & 0.73 & 786 & 1.15 \\
\hline Allemagne & 4.22 & 1181 & 3.49 & 838 & 1.21 \\
\hline France & 3.52 & 379 & 3.35 & 311 & 1.05 \\
\hline Italie & 1.32 & 360 & 1.21 & 237 & 1.09 \\
\hline Espagne, Portugal & 1.45 & 415 & 1.34 & 316 & 1.08 \\
\hline Autres pays UE/AELE & 3.81 & 716 & 3.44 & 696 & 1.11 \\
\hline Europe hors UE/AELE & 1.03 & 216 & 0.81 & 184 & 1.27 \\
\hline Autres pays OCDE & 8.65 & 270 & 7.21 & 288 & 1.20 \\
\hline Autres pays & 2.82 & 456 & 2.20 & 440 & 1.28 \\
\hline Total & 2.14 & 4587 & 1.69 & 4096 & 1.27 \\
\hline
\end{tabular}

Exemple de lecture : parmi la population allemande née à l'étranger et de sexe masculin, la probabilité de quitter la Suisse est de 4,22 \%, indiquant que pour 100 hommes présents en début d'année, quatre ont quitté la Suisse avant la fin de l'année.

La migration concerne essentiellement des jeunes adultes (Pecoraro, 2012 ; Nekby, 2006). C'est en effet aux jeunes âges (vingt-cinq/trentre-quatre ans 
pour les natifs de la Suisse, dix-huit/vingt-quatre ans pour les personnes nées à l'étranger) que la probabilité de migrer est la plus élevée. Cette probabilité diminue progressivement jusqu'à quarante-cinq/cinquante-quatre ans, pour s'accroître ensuite légèrement parmi les cinquante-cinq/soixante-quatre ans nés à l'étranger et se stabiliser chez les natifs de la Suisse. La probabilité est la plus faible après soixante-cinq ans. Les odds ratios confirment le rôle de l'âge avec, comparativement au groupe de référence (vingt-cinq/trentre-quatre ans), une baisse significative de l'odds ratio dès trente-cinq ans chez les natifs de la Suisse et les Suisses nés à l'étranger, et entre quarante-cinq et soixante-quatre ans chez les immigrés étrangers.

Pour la population née à l'étranger, les probabilités d'émigration varient d'une manière importante selon la nationalité : elles sont inférieures à $1 \%$ pour les Européens non membres de I'UE/AELE (principalement des ressortissants des Balkans) contre près de $8 \%$ pour les ressortissants de pays non européens, membres de I'OCDE (principalement américains et canadiens). Comparativement aux Allemands, l'odds ratio est significativement plus faible, après contrôle des autres variables, pour les Européens du Sud, les Français, mais aussi les ressortissants des pays de I'OCDE hors Europe. II est plus élevé pour les ressortissants des pays européens non membres de I'UE/AELE et ceux des pays en développement (non membres de l'OCDE). Ces résultats concordent avec les conclusions de l'étude de Gundel et Peters (2008) concernant la migration de retour des immigrés en Allemagne, qui démontre la plus grande probabilité de retourner dans leur pays pour les ressortissants d'États ayant des accords de libre circulation avec le pays d'accueil. La libre circulation des personnes semble également, dans le cas de la Suisse, influencer la probabilité de retour pour les groupes concernés. Par contre, les ressortissants de pays Européens n'ayant pas signé d'accords de libre circulation avec la Suisse émigrent moins souvent, certainement en raison de la difficulté, voire l'impossibilité, de revenir en Suisse après un séjour à l'étranger. Cependant, les résultats confirment également I'hypothèse de Constant et Massey (2003) sur le rôle du pays d'origine : les ressortissants des États membres de l'OCDE se caractérisent par une probabilité élevée de retour comparativement aux ressortissants des États non membres, probablement car les conditions de retour sont plus favorables et offrent plus d'opportunités d'insertion sur le marché du travail ; pour les citoyens d'Amérique du Nord, les conditions salariales et le pouvoir d'achat dans le pays d'origine doivent être proches de ceux observés en Suisse, ce qui peut favoriser le retour.

Pour Dustmann et Weiss (2007), ce sont les différentiels de coûts de la vie (et donc de pouvoir d'achat) entre le pays d'origine et d'accueil qui inciteraient les migrants à rentrer chez eux. Toutefois, on notera que cette hypothèse n'est pas vérifiée dans le cas suisse, puisque les ressortissants des États les plus pauvres présentent une probabilité de retour inférieure à celle des pays riches. L'hypothèse du différentiel de pouvoir d'achat à l'avantage du pays d'origine ne résiste pas à d'autres facteurs liés aux conditions de vie, de travail ou de sécurité, qui elles sont plutôt à l'avantage du pays d'accueil.

Il est probable, également, que le parcours migratoire des ressortissants des pays anglophones hors Europe soit plus souvent marqué par une forte circularité (allers-retours fréquents, déplacement dans plusieurs pays) caractéristique des cadres des multinationales ou des fonctionnaires internationaux : 
pour beaucoup de ces ressortissants, la présence en Suisse est alors considérée comme une étape temporaire dans le parcours professionnel, d'où une forte mobilité.

Parmi les natifs de la Suisse, l'origine étrangère (nationalité étrangère ou naturalisation au cours de la vie) accroît la probabilité de quitter la Suisse, comparativement aux personnes d'origine suisse. Ce résultat n'est pas surprenant compte tenu des liens qui peuvent toujours les unir à un pays d'origine. En outre, les natifs de la Suisse ayant connu une expérience migratoire durant leur vie ont un risque quatre fois plus élevé de quitter la Suisse. Pour ces personnes, le taux d'émigration se situe à $1,2 \%$, soit à mi-chemin entre celui des natifs de la Suisse sans expérience migratoire $(0,25 \%)$ et celui des natifs de l'étranger $(1,9 \%)$.

Pour la population née à l'étranger et de nationalité étrangère, la probabilité d'émigrer diminue à mesure que se prolonge la durée de séjour en Suisse. Cette probabilité est de $7,4 \%$ au cours de la première année de séjour ${ }^{13}$, mais de $0,8 \%$ seulement après dix ans, soit un niveau qui reste supérieur à celui des natifs de la Suisse (0,3\%), tout en s'y approchant. La stabilisation après cinq ans, observée par Schroll (2009) au Danemark, n'est pas avérée en Suisse. Les résultats obtenus confirment ceux mis en évidence par Pecoraro (2012), qui rattache la baisse de la probabilité d'émigrer au fil du temps à une meilleure intégration à la société d'accueil.

La probabilité élevée de départ dans les premières années qui suivent I'arrivée pourrait confirmer l'hypothèse de " mistaken migrants " de Constant et Massey (2003). Cette théorie fait référence aux immigrés qui ne rencontrent pas la situation prévue dans le pays hôte et décident donc de rentrer au pays assez rapidement. Cet échec migratoire s'expliquerait par un déficit d'informations au moment de la migration et des erreurs d'appréciation quant à la possibilité de s'intégrer dans un nouveau pays (OCDE, 2008). Cependant, nos analyses indiquent que les niveaux d'émigration avant cinq ans et les rythmes de diminution de ces quotients sont beaucoup plus élevés au sein de la population titulaire d'un diplôme tertiaire que de celle titulaire d'un diplôme du secondaire I. Si la théorie de Constant et Massey (2003) se vérifie, cela revient à dire que les titulaires d'un diplôme universitaire rencontrent une situation dans le pays d'accueil ne répondant plus souvent pas aux exigences requises, comparativement aux personnes faiblement ou moyennent qualifiées. Plus vraisemblablement, dans le cas suisse, $d$ 'autres facteurs sont susceptibles d'intervenir sur une migration de courte durée, tels que la disponibilité de stages professionnels ou la présence en Suisse de multinationales faisant circuler leurs élites. D'où une mobilité relativement importante liée à la configuration du marché du travail ainsi qu'aux opportunités professionnelles à l'étranger, plutôt qu'à des échecs migratoires (Tableau 3).

13 Rappelons que sont interrogées par le relevé structurel des personnes étrangères bénéficiant d'un permis de séjour annuel ou d'établissement, ainsi que celles ayant résidé en Suisse depuis au moins douze mois. Sont exclus de I'échantillon les étrangers bénéficiant d'un permis de courte durée. Ainsi, la probabilité de retour de $7,4 \%$ s'applique à des étrangers résidant en Suisse depuis moins d'un an tout en ayant la possibilité d'y rester au moins un an. 
Tableau 3 : Quotients d'émigration, selon le niveau de formation et la durée de séjour en Suisse pour les émigrant.e.s né.e.s à l'étranger, Suisse, 2011-2013

\begin{tabular}{lcccccc}
\hline & \multicolumn{2}{c}{ Secondaire I } & \multicolumn{2}{c}{ Secondaire II } & \multicolumn{2}{c}{ Tertiaire } \\
\cline { 2 - 7 } & $\%$ & $\mathrm{~N}$ & $\%$ & $\mathrm{~N}$ & $\%$ & $\mathrm{~N}$ \\
\hline$<1$ an & 4.99 & 130 & 6.85 & 216 & 8.69 & 642 \\
$1->5$ ans & 2.57 & 475 & 4.07 & 824 & 6.91 & 2606 \\
$5-<10$ ans & 1.43 & 308 & 1.73 & 284 & 2.92 & 676 \\
10 ans+ & 0.71 & 607 & 0.67 & 405 & 1.04 & 379 \\
Total & 1.25 & 1520 & 1.83 & 1729 & 4.17 & 4303 \\
\hline
\end{tabular}

Exemple de lecture : parmi la population de niveau secondaire I présente en Suisse depuis moins d'années, la probabilité de quitter la Suisse est de 4,99\%, indiquant que pour 100 personnes présentes en début d'année, cinq ont quitté la Suisse avant la fin de l'année.

Autant chez les natifs que chez les migrants, la probabilité d'émigrer est la plus élevée parmi les personnes vivant seules, un résultat qui peut s'expliquer par les moindres attaches en Suisse de ces personnes, comparativement aux ménages familiaux, confirmant le rôle de la composition familiale sur le mouvement migratoire mis en évidence par d'autres auteurs tels Faist (2008) et Boyd (1989). Chez les natifs de la Suisse, la probabilité de migrer est la plus faible dans les ménages familiaux sans enfant, alors que parmi les migrants, les ménages avec enfants sont les moins mobiles. Dans le premier groupe, les écarts entre ménages avec ou sans enfants ne sont pas significatifs après contrôle des autres variables incluses dans le modèle de régression logistique. Chez les migrants, les écarts sont significatifs uniquement pour les Suisses nés à l'étranger, mais pas pour les migrants étrangers. Cette absence de significativité contredit les résultats obtenus pour le début du XXle siècle en Suisse par Pecoraro (2012) et en Allemagne par Constant et Massey (2003), montrant le frein que constituent les enfants dans le projet migratoire, en particulier lorsqu'ils sont scolarisés. Cependant, l'impossibilité de contrôler l'âge des enfants dans notre modèle peut expliquer la différence entre les résultats obtenus ici et la littérature : en effet, une émigration n'a pas le même sens lorsque le ménage comprend des enfants non scolarisés ou des enfants en âge scolaire.

Le statut d'activité à la date du relevé structurel joue un rôle important sur la probabilité de quitter la Suisse. Parmi les natifs de la Suisse, une dichotomie très nette s'observe entre chômeurs/étudiants/ " autres " d'une part, présentant la probabilité d'émigration la plus élevée, et actifs/rentiers/au foyer d'autre part, qui présentent de plus faibles niveaux de départ. Ces résutats ne sont pas surprenants et confirment ceux observés par Bijwaard et Wang (2016) à partir de données néerlandaises, car l'insertion dans une activité professionnelle freine le départ alors que l'absence d'emploi l'accélère ; par ailleurs, les rentiers (veufs, invalides ou âgés) effectuent moins de mouvements migratoires que les actifs, un résultat qui s'explique certainement par la position dans le cycle de vie (fin de la vie active ou retraite) qui ne favorise pas le départ, et peut-être aussi par des raisons liées à l'état de santé. Enfin, se déclarer personne " au foyer " implique le plus souvent la présence dans le ménage d'un conjoint actif, qui influence certainement le choix de la personne interrogée quant à l'émigration. En 
revanche, les trois catégories présentant les plus hauts niveaux de mobilité sont aussi celles où les attaches professionnelles sont moindres : la fin des études ou le fait de ne pas avoir de travail peuvent être des facteurs favorisant l'émigration.

Chez les migrants, des résultats similaires s'observent, si l'on excepte un risque important de quitter la Suisse pour les personnes se déclarant sans activité professionnelle (personnes " au foyer ", principalement des femmes). Parmi les femmes au foyer figurent certainement des migrantes secondaires, ayant suivi leur mari au moment d'une migration professionnelle, et qui n'ont pas pu ou pas voulu s'intégrer sur le marché du travail : I'absence d'intégration professionnelle peut représenter un échec migratoire et favoriser le départ du ménage ; en même temps, l'absence d'insertion professionnelle pour ses femmes, et par conséquent le fait de se retrouver au foyer, peut refléter une migration de courte durée, pour laquelle la recherche d'un emploi n'est pas une priorité. Ces deux situations justifient alors une mobilité accrue.

Le quotient d'émigration élevé des chômeurs et des personnes au foyer renvoie à la théorie de la migration sélective de Borjas et Bratsberg (1994). Cette théorie se focalise sur la réémigration (de retour ou en direction d'un pays tiers) et distingue deux raisons de quitter le pays d'acceuil : la première regroupe les migrants qui ont réalisé les objectifs (économiques, ou de formation) prédéfinis de la migration, et qui rentrent une fois ces objectifs atteints : les motivations du retour pour ce groupe font plutôt référence aux facteurs pull de Lee (1966) ; la seconde concerne les migrants ayant échoué en raison d'informations erronées sur les gains potentiels liés à la migration et qui, pour reprendre la formulation de Lee (1966), sont influencés par les facteurs répulsifs. En conséquence, les migrants qui choisissent de rester dans un pays passent une double sélection ; la sélection d'entrée - la migration étant plus aisée pour ceux qui en ont les moyens physiques, humains ou économiques - et la sélection de sortie, puisque sont repoussés ceux qui échouent. Les auteurs relèvent également la meilleure information des immigrés, qui connaissent à la fois la situation de vie dans le pays d'origine et le pays d'accueil, et qui peuvent dès lors décider plus correctement du choix de rester ou de quitter le pays (pour rentrer) que les natifs, censés ne pas connaître les deux situations.

Ainsi, les personnes au chômage ou au foyer présentes dans nos données, étant confrontées à des conditions ne permettant pas de réaliser leur projet migratoire, retournent au pays : ils subissent donc, selon les termes de Borjas et Bratsberg (1994), une sélection à la sortie. Constant et Massey (2003) notent également qu'un point essentiel dans la migration de retour est le fait d'avoir ou non un emploi dans le pays d'accueil. Le fait de ne pas pouvoir s'intégrer au marché du travail pourrait également expliquer la forte émigration des chômeurs nés en Suisse. Lee (1966) suggère que ces personnes, influencées par des facteurs répulsifs, seraient sélectionnées négativement en termes de niveau de formation. Cela peut en effet se vérifier dans le tableau 4, comparant le niveau de formation des personnes ayant émigré, selon le statut professionnel. Le chômage, qui est l'exemple type de facteur répulsif, concerne des émigrants parmi lesquels les personnes de faible niveau de qualification sont surreprésentées (35\% des chômeurs sont des personnes de formation secondaire I alors que ce niveau ne représente que $21 \%$ de l'échantillon), comparativement aux autres catégories professionnelles (rentiers exceptés). En revanche, les personnes 
actives, qui quitteraient la Suisse plutôt en raison de l'attractivité d'autres pays, se caractérisent par une surreprésentation des personnes du niveau tertiaire.

Tableau 4 : Distribution du niveau d'éducation selon le statut d'activité pour les émigrant.e.s né.e.s à l'étranger, Suisse, 2011-2013

\begin{tabular}{lcccccc}
\hline & \multicolumn{2}{c}{ Secondaire I } & \multicolumn{2}{c}{ Secondaire II } & \multicolumn{2}{c}{ Tertiaire } \\
\cline { 2 - 7 } & $\%$ & $\mathrm{~N}$ & $\%$ & $\mathrm{~N}$ & $\%$ & $\mathrm{~N}$ \\
\hline Actif & 15.3 & 786 & 24.0 & 1195 & 60.7 & 3306 \\
Au chômage & 35.4 & 237 & 23.5 & 172 & 41.2 & 315 \\
Étudiant & 18.7 & 122 & 34.1 & 235 & 47.2 & 330 \\
Au foyer & 30.5 & 220 & 21.5 & 170 & 48.0 & 384 \\
Rentier AVS/AI & 45.5 & 285 & 27.7 & 174 & 26.8 & 169 \\
Autre & 24.6 & 66 & 29.2 & 79 & 46.2 & 119 \\
Total & 21.2 & 1716 & 25.0 & 2025 & 53.8 & 4623 \\
\hline
\end{tabular}

D’une manière générale, les probabilités d'émigration sont élevées pour les personnes détenant un niveau de formation tertiaire. Les écarts de risque vont du simple au double après contrôle des facteurs de confusion, et sont donc similaires à ceux mesurés par Pecoraro (2012). Ce résultat confirme les observations émises par de nombreux auteurs (Borjas et Bratsberg, 1994 ; Reagan et Olsen, 2000 ; Nekby, 2006 ; Haug 2012). II fait référence aux plus nombreuses opportunités professionnelles existant à l'étranger pour les personnes hautement qualifiées. En outre, l'émigration a un coût, non seulement économique, mais aussi humain (par exemple, le fait de quitter sa famille ou son environnement), pas chiffrable, mais perceptible par les candidats au départ, coût qui influence le choix de quitter ou non la Suisse. Les bénéfices, en termes salariaux, sont plus importants pour les personnes de niveau de formation tertiaire, et peuvent donc dépasser plus aisément le coût perçu et favoriser l'émigration dans le cadre d'une prise de décision rationnelle.

Le fait de résider dans une agglomération proche de la frontière n'est pas un élément déterminant dans le risque d'émigrer pour la population de nationalité étrangère née à l'étranger, mais l'est pour la population née en Suisse ainsi que pour les Suisses nés à l'étranger. La proximité d'une frontière semble dès lors jouer un rôle plus important pour les Suisses comparativement aux étrangers, ce qui s'explique probablement par le fait qu'elle intervient sur la connaissance des opportunités résidentielles dans le pays voisin.

De manière similaire, vivre dans une commune rurale ou urbaine n'a que peu d'impact sur le risque d'émigrer pour la population née à l'étranger : l'odds ratio, 0.9 , est cependant significatif, indiquant un risque légèrement plus faible de quitter la Suisse pour les résidents des communes rurales. Pour les personnes nées en Suisse, le fait de vivre en milieu rural diminue significativement la probabilité de départ. Ces résultats, qui contredisent Pecoraro (2012), lequel avait montré une probabilité accrue d'émigration pour les étrangers résidant dans les communes rurales, sont difficiles à commenter étant donné que les structures socioprofessionnelles des régions urbaines et rurales diffèrent. Dès lors, c'est peut-être autant le contexte économique que le lieu de vie qui influence la probabilité de migrer. 
Être propriétaire de son logement diminue de manière significative le risque d'émigrer pour la population née en Suisse comme à l'étranger. Le fait d'être propriétaire indique une plus grande intégration dans le pays hôte, une envie d'y résider à long terme et probablement également un succès dans la migration. En Suisse, où la proportion des propriétaires est faible en comparaison internationale, l'accès à la propriété traduit également un niveau de vie et des moyens financiers importants ; les crédits hypothécaires sont également plus accessibles en cas de stabilité professionnelle. Ainsi, il n'est pas étonnant d'observer une relation entre acquisition du logement et mobilité internationale diminuée.

\section{Discussion}

Cette analyse a permis de vérifier quels sont les déterminants de l'émigration depuis la Suisse, et de montrer, entre autres, la similitude dans les facteurs économiques, démographiques et sociaux, intervenant dans le groupe des natifs et dans celui des migrants. Comme toute analyse statistique, elle se limite à vérifier l'impact statistique de certaines dimensions quantitatives et objectives, sans pouvoir saisir les motivations des personnes et les mécanismes conduisant au départ. Pour cette raison, les résultats observés doivent être confrontés à la littérature existante pour en tirer une interprétation. Dans cette discussion, nous relevons les résultats qui nous paraissent les plus intéressants au vu des théories de la migration. Auparavant, il convient de relever certaines limites des données utilisées.

En premier lieu, le relevé structurel, qui représente la base de cette analyse, interroge des personnes résidant d'une manière permanente en Suisse, donc domiciliées dans le pays pour au moins une année. Comme toute enquête, il peine cependant à atteindre les personnes les plus mobiles ou étant arrivées au cours des années qui précèdent. Ainsi, la mobilité est sous-estimée et les quotients d'émigration enregistrés à partir de l'enquête sont inférieurs à ceux calculés à partir de la population totale (Wanner et al., 2016).

Deuxièmement, l'enquête a été menée par questionnaire écrit ou en ligne et repose donc sur l'auto-déclaration des personnes enquêtées. Celles-ci peuvent être soumises à un biais de désirabilité sociale, et répondre d'une manière pas toujours objective aux questions. Ce biais concerne en particulier le statut professionnel. Le mode de collecte explique aussi certaines difficultés à interpréter des résultats, par exemple ceux concernant la modalité " autre " pour le statut d'activité. Le questionnaire ne permet par ailleurs pas de mesurer pleinement l'intégration au pays hôte. Ainsi, la maîtrise de la langue locale, les attaches familiales dans le pays d'origine et le pays d'accueil, mais également la fréquence des relations (voyages) dans le pays d'origine sont autant de facteurs qui peuvent influencer la décision de départ, facteurs pour lesquels nous n'avons pas d'informations. De plus, cette décision de partir ou de rester en Suisse repose également sur des préférences qui ne sont pas saisies.

Finalement, les informations socioéconomiques et démographiques saisies font référence à la date de l'enquête et non la date du départ. Des évènements peuvent survenir entre ces deux dates, par exemple un licenciement, une séparation, et agir sur la probabilité de quitter la Suisse sans que l'on puisse mesurer leur effet. 
Malgré ces limites, les données présentent de nombreux avantages, en particulier liés à la taille importante de l'échantillon qui permet une analyse précise de phénomènes à faible occurrence. Ainsi, les résultats obtenus représentent une contribution originale concernant les déterminants du phénomène migratoire, vu sous l'angle du retour au pays ou du départ des natifs.

Parmi les résultats obtenus, l'influence des variables liées au parcours migratoire sur la probabilité de quitter la Suisse confirme les résultats mis en avant par la littérature (Pecoraro, 2012 ; Constant et Massey, 2002 ; Klinthäll, 2006 ; Reagan et Olsen, 2000). Le fait d'être né en Suisse ou d'y résider depuis plus de cinq ans, ou encore l'absence d'expérience migratoire par le passé, sont des facteurs de sédentarité, révélateurs (pour les étrangers résidant depuis plusieurs années) d'une bonne intégration à la société d'accueil ou de ce que Klinthäll (2006) nomme un " effet d'acclimatation ". Pour les natifs de la Suisse, le fait d'avoir déjà vécu une expérience à l'étranger est un indicateur de la mobilité future. Ce résultat semble indiquer que la connaissance de pays tiers peut intervenir favorablement sur la réalisation d'un projet émigratoire et, par extension, que les personnes mieux informées émigrent plus souvent que celles n'ayant pas eu l'occasion de quitter le pays.

La propension plus forte au départ pour les personnes nées à l'étranger et arrivées récemment sur le territoire confirme pour sa part la théorie des " mistaken migrants" (Constant et Massey, 2003) : les immigrés ne rencontrant pas dans le pays hôte les conditions comblant leurs attentes effectuent rapidement un deuxième mouvement migratoire. Ce résultat semble également aller dans le sens du modèle de l'émigration sélective de Borjas et Bratsberg (1994; " the return migration selection model "), selon laquelle les immigrés effectuent une migration de retour dans leur pays d'origine en raison d'un échec migratoire (la situation rencontrée dans le pays hôte ne permet pas d'atteindre les objectifs visés par la migration). Dans ce cas, des facteurs répulsifs - pour emprunter la terminologie de Lee (1966) - semblent agir ; les migrants quittant la Suisse après un séjour de courte durée sont ainsi sélectionnés négativement en termes de qualification et de statut d'emploi, ce qui n'est pas vérifié par les données. En effet, parmi les personnes effectuant un séjour de courte durée en Suisse se trouve une part importante de titulaires d'un diplôme universitaire. La présence en Suisse de nombreux sièges d'entreprises multinationales peut conduire à une forme de mobilité de courte durée, susceptible d'expliquer un séjour limité dans le temps, sans qu'il n'y ait forcément un échec migratoire.

L'intégration ou non au marché du travail est également un facteur déterminant quant à la décision de quitter la Suisse. Ceci confirme à nouveau la théorie de Borjas et Bratsberg (1994) pour les personnes nées à l'étranger : lorsque le pays hôte ne fournit pas les conditions espérées, un nouveau départ s'impose. II est toutefois intéressant de noter que pour la population née en Suisse, l'absence d'intégration au marché du travail agit également comme un facteur " push ". S'il ne s'agit pas dans ce cas d'un échec migratoire, la possibilité de trouver de meilleures opportunités professionnelles à l'étranger semble jouer un rôle dans la décision de départ.

Par ailleurs, le fait de détenir un niveau d'éducation tertiaire est un des déterminants forts de l'émigration, confirmant les résultats de nombreux auteurs 
(Pecoraro, 2012 ; Zhao et al., 2000 ; Schroll, 2009 ; Nekby, 2006) : ce résultat indique que les facteurs répulsifs ne sont pas les seuls en cause, puisqu'une part de l'émigration s'explique probablement par des facteurs attractifs liés au pays de destination. Cette sélectivité quant au niveau de formation semble aussi indiquer que les personnes détenant un capital humain élevé ont un meilleur accès à l'information et donc de meilleures opportunités professionnelles à l'étranger.

Finalement, le cadre juridique de la migration semble également influencer fortement la décision de départ de la population née à l'étranger. Les pays de $I^{\prime} O C D E$ ainsi que de I'UE/AELE, pour lesquels les barrières administratives à la migration sont moindres, présentent une plus forte tendance au départ. La situation s'inverse lorsque l'on considère les pays européens hors UE/AELE. II est toutefois difficile de déduire des données disponibles si ce sont ces barrières à la migration ou la situation économique, voire sécuritaire, du pays d'origine qui influence la décision de départ.

II n'est pas possible d'identifier un seul profil type de l'émigrant, tant les motifs influençant le départ sont variés. Le modèle de régression logistique montre que l'émigration est la plus fréquente parmi les hommes jeunes, célibataires, nés à l'étranger, ressortissants d'un pays de l'OCDE, et arrivés récemment en Suisse, détenant un niveau de formation tertiaire, et en formation ou sansemploi. Cependant, la décision migratoire semble complexe et dépend autant de facteurs individuels liés au parcours de vie (âge, genre, situation familiale) et à l'intégration dans le pays d'accueil (durée de séjour, situation sur le marché du travail, capital humain), que de facteurs contextuels liés à la situation économique du pays d'origine ou aux barrières administratives à la migration. Elle peut être provoquée par des facteurs répulsifs, telle une situation de chômage, par des facteurs attractifs, liés aux opportunités professionnelles dans un autre pays, mais le plus souvent, certainement, par une conjonction de ces deux dimensions. La littérature internationale met en évidence des effets de genre, des effets liés à la réalisation des objectifs du séjour ou encore aux échecs migratoires pour expliquer l'émigration. Nos données ne permettent pas de valider totalement ces propositions, probablement car les motivations de l'émigration sont trop complexes pour rentrer dans un nombre limité de propositions théoriques.

D'une manière générale, si l'intensité de l'émigration varie, les résultats obtenus sont proches quel que soit le statut migratoire (personne née en Suisse, migrant de nationalité étrangère, Suisse né à l'étranger). La seule spécificité, concernant les migrants de nationalité étrangère, fait référence à la fréquence de la migration de retour en fin de vie active. Si l'on excepte cette spécificité, la similitude des résultats obtenus par les différents modèles suggère que les déterminants de l'émigration sont universels et ne dépendent pas du statut migratoire.

En conclusion, rappelons que les données se réfèrent à un pays, la Suisse, qui se caractérise par des flux migratoires en constante progression, favorisés par une situation conjoncturelle meilleure que dans les pays voisins. On peut s'interroger sur les motivations du départ d'un pays présentant un avantage comparatif en termes d'emplois. Les niveaux et les déterminants de la migration 
de retour sont évidemment influencés par les caractéristiques économiques, politiques et sociales du pays d'accueil. Cependant, la mobilité internationale répond à des logiques spécifiques qui ne sont pas différentes en Suisse et ailleurs : l'émigration ne fait pas forcément référence à des considérations purement rationnelles ou d'optimisation des profits, mais aussi au vécu, à la famille, et aux aspirations. Ainsi, les déterminants de l'émigration restent les mêmes quel que soit le contexte dans lequel la personne vit. Nos résultats confirment dès lors ceux obtenus dans d'autres pays industrialisés.

\section{Références bibliographiques}

Bachmeier James, Lessard-Phillips Laurence and Fokkema Tineke (2013) The gendered dynamics of integration and transnational engagement among second-generation adults in Europe, in Laura Oso and Natalia Ribas Mateos Eds., International Handbook on Gender, Migration and Transnationalism, Cheltenham (United Kingdom), Edward Elgar Publishing, pp. 268-293.

Bijwaard Govert E. and Wang Qi (2016) Return Migration of Foreign Students, European Journal of Population, 32 (1), pp. 31-54.

Borjas George and Bratsberg Bernt (1994) Who Leaves? The Outmigration of the Foreign-Born, The Review of Economics and Statistics, 78 (1), pp. 165-176.

Boyd Monica (1989) Family and Personal Networks in International Migration: Recent Developments and New Agendas, International Migration Review, 23 (3), pp. 638-670.

Constant Amelie and Massey Douglas (2003) Self-Selection, Earnings, and out-Migration: A Longitudinal Study of Immigrants to Germany, Journal of Population Economics, 16 (4), pp. 631-53.

Constant Amelie and Massey Douglas (2002) Return Migration by German Guestworkers: Neoclassical versus New Economic Theories, International Migration, 40 (4), pp. 5-38.

Cox David R. and Snell E.J. (1989) Analysis of Binary Data. Second Edition, USA, CRC Press, $240 \mathrm{p}$.

Dustmann Christian and Weiss Yoram (2007) Return migration: Theory and empirical evidence from the UK, British Jounrla of Industrial Relations, 45 (2), pp. 236-256.

Faist Thomas (2008) Migrants as Transnational Development Agents: An Inquiry Into the Newest Round of the Migration-Development Nexus, Population, Space and Place, 14 (1), pp. 21-42.

Gundel Sebastian and Peters Heiko (2008) What Determines the Duration of Stay of Immigrants in Germany?, International Journal of Social Economics, 35 (11), pp. 769-782.

Haug Sonja (2012) Migration and return migration: the case of Italian migrants in Germany, Kakanienrevisited, 26/07/2012, pp. 1-11

Klinthäll Martin (2006) Immigration, Integration and Return Migration, International Symposium on International Migration and Development, Turin, pp. 28-30. 
Lee Everett S. (1966) A Theory of Migration, Demography, 3 (1), pp. 45-47.

Nekby Lena (2006) The Emigration of Immigrants, Return vs Onward Migration: Evidence from Sweden, Journal of Population Economics, 19 (2), pp. 197-226.

OCDE (2008) Partie III, Les migrations de retour : un nouveau regard, in OCDE Éd., Perspectives des migrations internationales, Paris, OCDE, SOPEMI, pp. 181-246.

Pecoraro Marco (2012) Rester ou partir : les déterminants de l'émigration hors de Suisse, in Philippe Wanner, Ilka Steiner, Marco Pecoraro, Mathias Lerch etTopgul Ceren, La démographie des étrangers en Suisse, Genève, Seismo, pp. 141-155.

Reagan Patricia B. and Olsen Randall J. (2000) You Can Go Home Again: Evidence from Longitudinal Data, Demography, 37 (3), pp. 339-350.

Schroll Sanne (2009) Emigration of Immigrants: A Duration Analysis, Copenhage, University Press of Southern Denmark, $40 \mathrm{p}$.

Steiner Ilka and Wanner Philippe (2015) Towards a new data set for the analysis of migration and integration in Switzerland, Working Paper $\mathrm{n}^{\circ} 1$, Neuchâtel, NCCR On the Move.

Wanner Philippe, Zufferey Jonathan and Fioretta Juliette (2016) The Impact of Migratory Flows on the Swiss Labour Market. A Comparison Between in- and Outflows, Migration Letters, 13 (3) pp. 411-426.

Zhao John, Drew Doug and Murray Scott T. (2000) Brain Drain and Brain Gain: The Migration of Knowledge Workers from and to Canada, Education Quarterly Review, 6 (3), pp. 8-35.

\section{Annexe}

Tableau 5 : Description des variables introduites dans le modèle

\begin{tabular}{lcccc}
\hline & \multicolumn{2}{c}{ Nés en Suisse } & \multicolumn{2}{c}{ Nés à l'étranger } \\
\cline { 2 - 5 } & $\%$ & $\mathbf{N}$ & $\%$ & $\mathbf{N}$ \\
\hline Total & $\mathbf{1 0 0 . 0}$ & $\mathbf{5 8 4} \mathbf{5 8 2}$ & $\mathbf{1 0 0 . 0}$ & $\mathbf{2 3 7} \mathbf{7 5 8}$ \\
Genre & & & & \\
Homme & 49.1 & 282261 & 48.3 & 112876 \\
Femme & 50.9 & 302321 & 51.7 & 124882 \\
Âge & & & & \\
18-24 & 12.0 & 66266 & 6.9 & 14039 \\
$\mathbf{2 5 - 3 4}$ & 15.1 & 86061 & 21.1 & 46923 \\
$\mathbf{3 5 - 4 4}$ & 16.7 & 99185 & 23.1 & 54023 \\
$\mathbf{4 5 - 5 4}$ & 18.8 & 110228 & 21.2 & 51677 \\
$55-64$ & 15.8 & 93388 & 12.9 & 32385 \\
$65+$ & 21.6 & 129454 & 14.9 & 38711
\end{tabular}


Pays de nationalité

Suisse

$33.1 \quad 85691$

Allemagne

$12.3 \quad 26917$

France

$9.4 \quad 24137$

Italie

$4.2 \quad 10931$

Espagne/Portugal

$11.2 \quad 27049$

Autres pays de I'UE/AELE

$9.0 \quad 19821$

Europe hors UE/AELE

Hors Europe pays de I'OCDE

$10.9 \quad 21489$

Hors Europe non membres de I'OCDE

$1.7 \quad 3787$

Nationalité

Suisse depuis la naissance

$8.3 \quad 17910$

Naturalisé

Étranger

$\begin{array}{cccc}92.1 & 536946 & 5.1 & 13027 \\ 4.9 & 31003 & 27.5 & 71127 \\ 3.0 & 16559 & 67.3 & 152042 \\ & & & \\ & & 4.3 & 6986 \\ & & 24.6 & 40150 \\ & & 18.6 & 31933 \\ & & 52.5 & 95755\end{array}$

Durée du séjour en Suisse

Moins de 1 an

$1-<5$ ans

$5-<10$ ans

$52.5 \quad 95755$

Expérience migratoire au cours de la vie

Non

Oui

Type de ménage

Ménage individuel

Ménage familial sans enfant

Ménage familial avec enfant(s)

Statut d'activité

Actif

Au chômage

Étudiant

Au foyer

AVS/AI

Autre

Niveau de formation

Secondaire I

Secondaire II

Tertiaire

Proximité de la frontière

Autre agglomération

Agglomération frontalière

Lieu de résidence

Commune urbaine

Commune rurale

Statut du logement

Locataire

Propriétaire
96.4563140

$3.6 \quad 21442$

$\begin{array}{cccc}24.2 & 117811 & 23.4 & 44010 \\ 34.0 & 167217 & 29.4 & 56827 \\ 41.8 & 204381 & 47.2 & 88913 \\ & & & \\ 65.2 & 376498 & 64.4 & 150337 \\ 2.1 & 12564 & 5.4 & 12545 \\ 3.6 & 21208 & 2.7 & 6179 \\ 4.2 & 25450 & 7.2 & 16756 \\ 23.9 & 142825 & 18.4 & 47017 \\ 0.9 & 5424 & 1.8 & 4232\end{array}$

$\begin{array}{llll}17.7 & 102556 & 35.7 & 81479\end{array}$

$56.3 \quad 323512 \quad 33.3 \quad 75234$

$26.0 \quad 154895 \quad 31.0 \quad 73203$

$88.2 \quad 514672 \quad 79.9 \quad 187674$

$\begin{array}{llll}11.8 & 69910 & 20.1 & 50084\end{array}$

$69.9 \quad 409179 \quad 83.4 \quad 199034$

$\begin{array}{llll}30.1 & 175403 & 16.6 & 38724\end{array}$

$51.1 \quad 254656 \quad 73.5 \quad 140146$

$\begin{array}{llll}48.9 & 237760 & 26.5 & 52608\end{array}$

Source : Relevés structurels 2010-2012. 


\section{Juliette Fioretta et Philippe Wanner}

\section{Rester ou partir? Les déterminants des flux d'émigration récents depuis la Suisse}

La Suisse enregistre chaque année plus de 100000 départs en direction de l'étranger. L'importance de l'émigration surprend alors que la conjoncture économique dans le pays et le marché du travail présentent une situation favorable en comparaison internationale. À partir de données longitudinales comprenant plus de 800000 individus suivis sur trois années consécutives, cet article tente de comprendre qui sont les résidents quittant le pays, et plus particulièrement quels sont les facteurs intervenant dans le choix d'effectuer une émigration. Les résultats montrent que la décision migratoire est complexe et dépend autant de facteurs individuels liés au parcours de vie (âge, genre, situation familiale) et à l'intégration dans le pays d'accueil (durée de séjour, situation sur le marché du travail, capital humain), que de facteurs contextuels liés à la situation économique du pays d'origine ou aux barrières administratives à la migration. Elle peut être provoquée par des facteurs répulsifs, telle une situation de chômage, par des facteurs attractifs, liés aux opportunités professionnelles dans un autre pays, mais le plus souvent, certainement, par une conjonction de ces deux dimensions.

\section{Stay or Leave? Deciding Factors in the Recent Emigration Flow from Switzerland}

Every year, Switzerland records more than 100,000 departures to another country. The magnitude of this emigration flow is surprising, as the economic conjuncture and the labor market are in a favorable position in international comparison. Using longitudinal data of more than 800,000 individuals followed over three consecutive years, this article attempts to understand which residents are leaving the country, and more specifically which factors are involved in the decision to migrate. The results show that this decision is complex and depends as much on individual factors related to life course (age, gender, family status) and integration in the host country (residence time, situation on the labor market, human capital) as on contextual factors related to the economic situation of the country or administrative barriers to migration. Emigration can be favoured by push factors such as a situation of unemployment, or by pull factors related to career opportunities in another country; but most often, probably, by a combination of both. 


\section{Los determinantes de los flujos recientes de emigración desde Suiza}

Cada año en Suiza, se registran más de 100000 salidas del territorio. La importancia de este fenómeno no deja de sorprender tomando en cuenta que tanto la situación económica del país como la del mercado laboral, constituyen un ámbito favorable incluyéndolos en una perspectiva internacional. A partir de datos longitudinales que comprenden más de 800000 individuos seguidos durante tres años consecutivos, este artículo se propone entender quiénes son los residentes que emigran de Suiza, y sobre todo, cuales son los factores que están a raíz de su decisión de emigrar. Los resultados muestran la complejidad de esta decisión y su dependencia tanto en factores individuales ligados al curso de la vida (edad, sexo, situación familiar) y a la integración en el país de acogida (tiempo de residencia, situación en el mercado laboral, capital humano), como en factores contextuales relacionados con la situación económica del país de origen o con las barreras administrativas puestas antes la migración. Existen factores repulsivos que incitan a la emigración, como una situación de desempleo, pero también factores atractivos, como la existencia de oportunidades profesionales en otro país. Una combinación de ambos fenómenos vendría a ser con más frecuencia lo que determina la decisión de emigrar. 